\title{
The Influence of
}

\section{Extracellular Products on the Behaviour of Mixed Microbial Populations in Magnesium-limited Chemostat Cultures}

\author{
By J. L. MEERS AND D. W. TEMPEST \\ Microbiological Research Establishment, Porton, near Salisbury, Wiltshire
}

(Accepted for publication I8 January I968)

SUMMARY

Magnesium-limited mixed cultures, each containing two microbial species, were produced by exchange of small numbers of organisms between magnesium-limited chemostat cultures of Aerobacter aerogenes, Pseudomonas fluorescens, Bacillus subtilis, B. megaterium, Staphylococcus epidermis and Torula utilis. The fate of each species in these mixed cultures was determined following cross-inoculation. Gram-negative organisms invariably outgrew the Gram-positive ones, but the ability of $B$. subtilis and B. megaterium to outgrow each other, or to outgrow the yeast, depended on the inoculum size. This dependence resulted from the presence of specific extracellular products in the Bacillus cultures which stimulated their growth and uptake of magnesium. The concentration of these extracellular growth-promoting substance(s) in magnesium-limited cultures of B. subtilis varied with population density. Thus, when the magnesium concentration in the medium was decreased from 0.9 to $0.15 \mu \mathrm{g}$. $/ \mathrm{ml}$. (thereby causing the steady-state population density to be decreased to one-eightieth the initial value), $B$. subtilis could no longer maintain itself against proportionately low concentrations of $T$. utilis. But addition of extracellular fluids from dense magnesium-limited $B$. subtilis cultures to magnesium-limited $T$. utilis cultures enabled small numbers of the Bacillus now to outgrow the yeast. From these two observations it is concluded that magnesium assimilation by $B$. subtilis is more dependent on extracellular substance(s) than is magnesium assimilation by either T. utilis or the Gram-negative organisms. Modification of the basic theory of microbial growth in a chemostat culture, to take account of product-stimulated substrate-assimilation, is suggested.

\section{INTRODUCTION}

It is generally assumed (Monod, 1950; Herbert, Elsworth \& Telling, 1956; Powell, 1965) that the relationship between the growth rate of organisms and the concentration of growth-limiting nutrient, in a chemostat culture, can be represented adequately by a Michaelis-Menten type of equation:

$$
[D=] \mu=\mu_{\max }\left(\frac{s}{K_{s}+s}\right),
$$

where $\mu$ is the specific growth rate, $\mu_{\max }$ the maximum rate of growth of the organisms in the medium, $s$ the extracellular concentration of growth-limiting substrate in the culture $\left(\mathrm{Mg}^{2+}\right.$ in most of the experiments to be described here) and $K_{s}$ a saturation constant (numerically equal to the extracellular concentration of growth-limiting 
nutrient at $\left.0.5 \mu_{\max }\right)$. Under steady-state conditions in a chemostat, $\mu$ is equal to the dilution rate $(D)$.

In the above equation there are two variables $(\mu$ and $s)$ and when these are plotted against one another for a pure culture of an organism $(A)$, a curve is obtained as shown in Fig. I. With cultures of a different organism $(B)$ a plot of similar shape would be obtained, but it would not be identical to the curve for organism $A$ unless both of the constants $\left(\mu_{\max }\right.$ and $\left.K_{\mathrm{s}}\right)$ were also identical. Now, assuming the saturation curves for two organisms to be exactly as shown in Fig. I, and that these were grown in separate chemostats under identical (magnesium-limited) conditions at a dilution rate of $D_{1} \mathrm{hr}^{-1}$, then the extracellular concentration of $\mathrm{Mg}^{2+}$ in the culture of $A$-type organisms would be $s_{A}$, whereas that in the culture of $B$-type organisms would be $s_{B}$. If a small number of $A$-type organisms were now transferred to the chemostat containing a steady-state population of $B$-type organisms, then (assuming no lag period) $A$-type organisms would begin growing at a rate $\mu_{A}$ (since the growth-limiting substrate concentration would be $s_{B}$; Fig. I). Because $\mu_{A}$ is greater than $D_{1}$, the concentration of $A$-type organisms in the culture would increase. Their growth would cause the growth-limiting substrate concentration to decrease towards the value $s_{A}$; at this latter concentration $B$-type organisms could grow only at a rate $\mu_{B}$ which, being less than $D_{1}$, would result in them being washed completely from the culture. Thus, organism $A$ would totally displace organism $B$ from the chemostat culture and this should occur irrespective of the initial concentration of each organism in the mixed culture and, in this case, the dilution rate.

We described previously (Tempest, Dicks \& Meers, 1967) how Aerobacter aerogenes outgrew several Gram-positive organisms in $\mathbf{M g}^{2+}$-limited mixed culture experiments in a manner predicted by the above theory. But mixed cultures of Torula utilis and Bacillus subtilis behaved paradoxically: neither organism outgrew the other species unless the inoculum population exceeded a certain minimum concentration. The present paper reports the results of experiments designed to explain the unexpected behaviour of these mixed cultures. A preliminary report has been published (Meers \& Tempest, 1968).

\section{METHODS}

Organisms. Aerobacter aerogenes (NCTC 4I8); Pseudomonas fluorescens (strain KB I, from the University of Sheffield); Bacillus subtilis var. niger (ATCC 9372); B. megaterium (strain KM, from the University of Edinburgh); Staphylococcus epidermidis (isolated by Mr F. A. Dark, Porton). Each organism was maintained on tryptic meat digest agar slopes. Torula utilis (NCYC 321) was maintained on yeast extract peptone glucose agar slopes.

Growth conditions. Continuous cultures of organisms were maintained in 0.251 . chemostats (designed by Dr D. Herbert) without $\mathrm{pH}$ control. The magnesium-limited medium was basically that described previously (Tempest et al., 1967), but the magnesium content was varied as indicated in the Results section. The potassiumlimited medium was that described by Tempest, Dicks \& Hunter (I966), but with the potassium concentration decreased to $0.15 \mathrm{mM}$; the carbon-limited medium was that of Tempest, Hunter \& Sykes (1965) but with glucose (I g./1.) replacing the glycerol. The same magnesium-limited medium, with the magnesium concentration adjusted to $0.02 \mathrm{mM}$, was used for batch culture experiments. 
In some experiments the medium was supplemented with supernatant fluids from previous chemostat cultures. These fluids were obtained by rapid filtration of the culture through a sintered glass filter (no. E5), to eliminate most of the organisms, and then through a membrane filter (Oxoid; standard grade) to effect their complete removal. Where larger volumes of culture were to be treated, the bulk of organisms were removed by centrifugation ( $5000 \mathrm{~g}$ for $5 \mathrm{~min}$.), before sterilization by filtration as above.

The technique for growing mixed cultures of organisms in the chemostat was that described by Tempest et al. (1967). For batch culture experiments, $20 \mathrm{ml}$. volumes of medium (contained in $350 \mathrm{ml}$. conical flasks) were inoculated with organisms from magnesium-limited chemostat cultures and aerated by shaking, on a reciprocating shaker, at $37^{\circ}$. At appropriate intervals of time, $3 \mathrm{ml}$. samples were withdrawn from each culture and measurements made of the extinction at $540 \mathrm{~m} \mu$ (Bausch and Lomb 'Spectronic 20' spectrophotometer with tube of 0.5 in. ( $1.2 \mathrm{~cm}$.) internal diameter) and culture population density (by counting directly the number of organisms in a known volume of culture, by using a Thoma Haemocytomer of $0.02 \mathrm{~mm}$. depth). The results obtained by both methods correlated closely.

Magnesium determinations were made using an EEL (model I40) atomic absorption spectrophotometer on samples prepared as described previously (Tempest et al. 1967).

\section{RESULTS}

The magnesium-limited mixed culture experiments reported previously (Tempest et al. 1967) were extended to include most the possible combinations of two species from the following group of organisms: Aerobacter aerogenes, Pseudomonas fluorescens, Bacillus subtilis, B. megaterium, Staphylococcus epidermidis and Torula utilis. It was found that the Gram-negative bacteria invariably outgrew the Gram-positive organisms rapidly, irrespective of the initial population densities of the two competing species in the mixed cultures. Thus it can be concluded that both $A$. aerogenes and $P$. fluorescens have more efficient mechanisms for the uptake of magnesium from their environment than the Gram-positive organisms listed above. It is important to point out that S. epidermidis would grow only in simple salts media that had been supplemented with Casamino acids (I \%, w/v) and yeast extract $(0.001 \%, w / v)$, each of which contain substances that stimulated growth and the uptake of magnesium by $B$. subtilis (Tempest et al. 1967) and presumably also by the Staphylococcus organisms. Nevertheless, $S$. epidermidis cultures still were rapidly outgrown by either $A$. aerogenes or $P$. fluorescens organisms.

The ability of Bacillus megaterium and B. subtilis to outgrow each other or to outgrow Torula utilis in magnesium-limited mixed cultures depended on the relative amounts of each species in the culture immediately after mixing the two populations. Thus, when magnesium-limited mixed cultures of either B. megaterium and T. utilis or $B$. megaterium and $B$. subtilis were prepared, the ultimate fate of each species in each mixed culture depended on their concentrations immediately after crossinoculation (Fig. 2). These results, which were identical to that with B. subtilis and T. utilis (Tempest et al. 1967), cannot be predicted from the theory outlined in the Introduction, since equation (I) contains no factor for population density.

The most obvious explanation for these paradoxical take-over patterns is that the 
Gram-positive organisms produced substances that accumulated in the culture extracellular fluids and inhibited the growth of the competing species. To investigate this possibility, batch cultures of Bacillus subtilis, B. megaterium and Torula utilis were grown in low-magnesium simple salts media which had been supplemented with equal volumes of the supernatant fluids from magnesium-limited chemostat cultures of the three species. The resulting growth curves (Fig. 3) showed no evidence of growth inhibition. On the contrary, the growth curves indicated clearly the presence

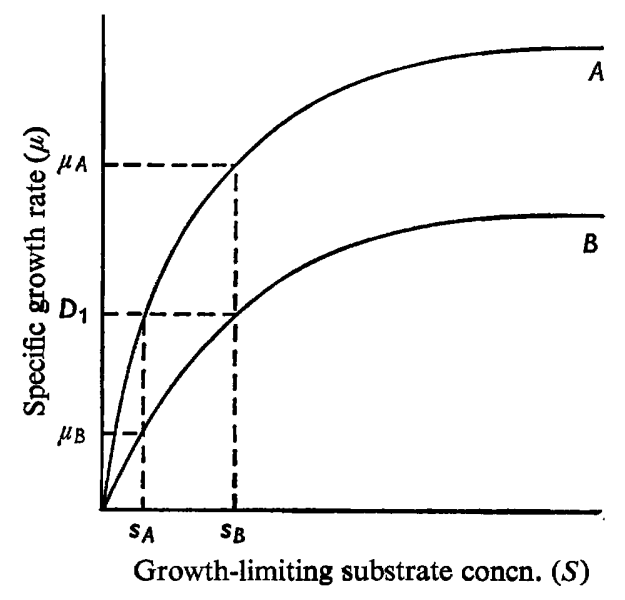

Fig. I

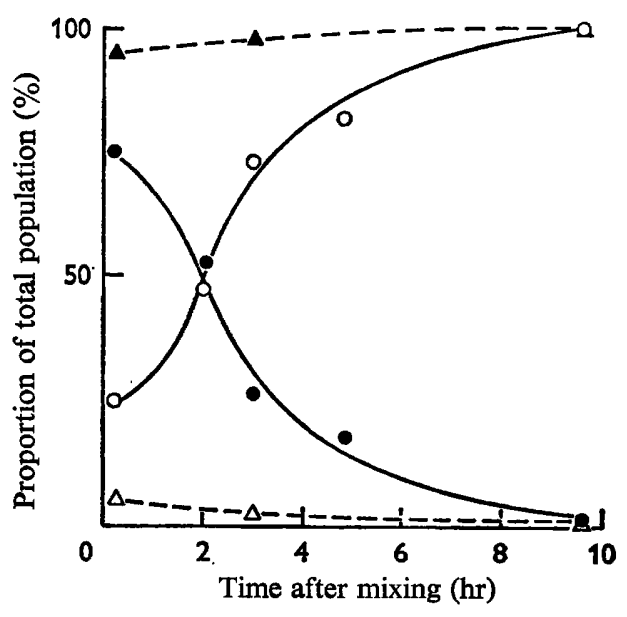

Fig. 2

Fig. 1. Hypothetical saturation curves for two microbial species ( $A$ and $B$ ) growing in magnesium-limited chemostat cultures. At a fixed dilution rate $\left(D_{1}\right)$ the growth-limiting substrate concentration in a culture of $A$ organisms would be $s_{A}$ and of $B$ organisms $s_{B}$. It is assumed that the maximum growth rate $\left(\mu_{\max }\right)$ for $A$ organisms is greater than for $B$ organisms and that the saturation constants ( $K_{s}$ values) bear a similar relationship.

Fig. 2. Growth of Bacillus megaterium and Torula utilis in magnesium-limited simple salts medium, in a chemostat. In the experiment represented by the solid lines the initial concentration of $B$. megaterium (O) was $9 \times 10^{7} \mathrm{orgs} / \mathrm{ml}$. and T. utilis (O) $3 \times 10^{7} \mathrm{orgs} / \mathrm{ml}$., i.e. $75 \%$ and $25 \%$ of the initial mixed population, respectively. Also recorded (broken lines) are the results of a similar experiment in which the initial concentration of $B$. megaterium ( $\Delta$ ) was increased to $\mathrm{I} \cdot 8 \times 10^{8}$ orgs $/ \mathrm{ml}$. and that of $T$. utilis $(\Delta)$ lowered to $\mathrm{I} \times 1 \mathrm{I}^{7}$ orgs/ ml., i.e. $95 \%$ and $5 \%$ of the total population, respectively. In both experiments the dilution rate was $0.3 \mathrm{hr}^{-1}$ and the temperature $33^{\circ}$. The $\mathrm{pH}$ value was not controlled but did not vary beyond the range $6 \cdot 1$ to $6 \cdot 5$ in each experiment.

of growth-promoting substances in the culture extracellular fluids. Some specificities were apparent since the growth of each of the Bacillus species was promoted to the greatest extent by its own extracellular fluid (Fig. 3). It seemed possible, therefore, that the effect of population density on the growth patterns of Gram-positive organisms in magnesium-limited mixed cultures resulted from the presence of specific substances, secreted into the culture fluids, which facilitated magnesium assimilation.

If the above hypothesis is correct, then addition of extracellular fluid from a magnesium-limited Bacillus culture to the medium supplying a mixed culture of that Bacillus species and, for example, yeast organisms, possibly could enable small numbers of the bacilli now to outgrow the yeast. When sterile supernatant fluid from a magnesium-limited Bacillus subtilis culture which had contained $5 \times 10^{8}$ bacilli $/ \mathrm{ml}$. 
was mixed $(50 \%, \mathrm{v} / \mathrm{v})$ with a magnesium-limited simple salts medium $\left(0.9 \mu \mathrm{g} . \mathrm{Mg}^{2+}\right)$ $\mathrm{ml}$., final concentration) and used as the medium supplying a magnesium-limited chemostat culture containing Torula utilis $\left(4.5 \times 10^{7}\right.$ organisms $/ \mathrm{ml}$.) and B. subtilis $\left(2 \times 10^{6}\right.$ bacilli/ml., initial concentration), the bacilli now outgrew the yeast organisms (Table 1 ).

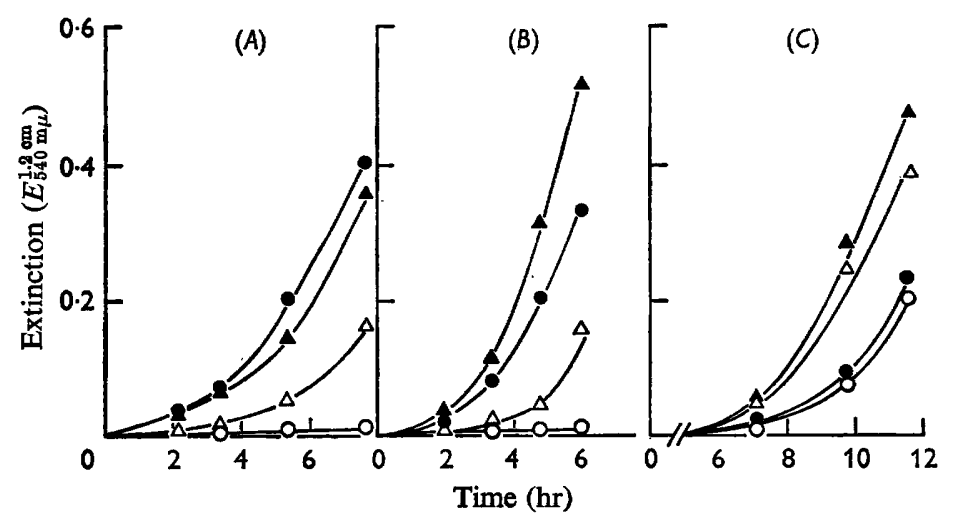

Fig. 3

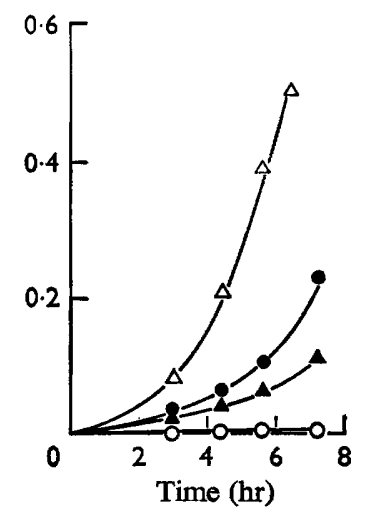

Fig. 4

Fig. 3. Growth of $(A)$ Bacillus subtilis, $(B)$ Bacillus megaterium and $(C)$ Torula utilis in simple salts media that were supplemented $(50 \%, v / v)$ with cell-free fluid from magnesiumlimited cultures of $B$. subtilis $(\mathbf{O}), B$. megaterium $(\Delta)$ and $T$. utilis $(\triangle)$; growth of organisms in an unsupplemented medium is shown by the open circles. The cultures were grown in $350 \mathrm{ml}$. conical flasks, incubated at $37^{\circ}$ on a reciprocating shaker. All the media contained $0.5 \mu \mathrm{g} . \mathrm{Mg}^{2+} / \mathrm{ml}$. and in each case the inoculum was from a magnesium-limited chemostat culture of the appropriate organism.

Fig. 4. Growth of Bacillus subtilis in a simple salts medium containing $0.5 \mu \mathrm{g} . \mathrm{Mg}^{2+} / \mathrm{ml}$. that had been $(\triangle)$ supplemented $(50 \%, v / v)$ with cell-free fluid from a magnesium-limited culture of $B$. subtilis which had contained about $5 \times 10^{8} \mathrm{orgs} / \mathrm{ml}$., (A) supplemented $(5 \%, \mathrm{v} / \mathrm{v})$ with the same cell-free fluid, and $(\odot)$ supplemented $(50 \%, \mathrm{v} / \mathrm{v})$ with cell-free fluid from a magnesium-limited $B$. subtilis culture that had contained about $1 \times 10^{8} \mathrm{orgs} / \mathrm{ml}$. The open circles refer to growth of $B$. subtilis in an unsupplemented medium. The cultures were grown in $350 \mathrm{ml}$. conical flasks, incubated at $37^{\circ}$ on a reciprocating shaker. The inoculum was from a magnesium-limited chemostat culture $\left(D=0.3 \mathrm{hr}^{-1}, 33^{\circ}, \mathrm{pH} 6.5\right)$.

\section{Table I. Growth of Bacillus subtilis and Torula utilis in a magnesium-limited chemostat} culture supplemented with magnesium-limited B. subtilis extracellular culture fluid

The culture temperature was controlled at $33^{\circ}$; the $\mathrm{pH}$ value was not controlled but varied only from $6 \cdot 1$ to 6.4 during the experiment. The magnesium-limited simple salts medium was mixed $(50 \%, v / v)$ with cell-free fluid from a magnesium-limited B. subtilis culture which had contained about $5 \times 10^{8}$ bacilli $/ \mathrm{ml}$.; the total magnesium concentration was adjusted to $0.9 \mu \mathrm{g}$. $/ \mathrm{ml}$. The dilution rate was fixed at $0.3 \mathrm{hr}^{-1}$.

\begin{tabular}{cccccc} 
Time after & No. of orgs/mi. $\left(\times 10^{-7}\right)$ & \multicolumn{2}{c}{$\begin{array}{c}\text { Proportion of } \\
\text { total no. of orgs (\%) }\end{array}$} \\
\cline { 2 - 3 } mixing (hr) & B. subtilis & T. utilis & & B. subtilis & T. utilis \\
0 & 0.2 & 4.5 & 5 & 95 \\
3 & 0.3 & 5.6 & 5 & 95 \\
8 & 0.7 & 5.0 & 12 & 88 \\
13 & 4.6 & 5.0 & 48 & 52 \\
21 & 13.2 & $2 \cdot 2$ & 86 & 14 \\
26 & 20.0 & $1 \cdot 8$ & 92 & 8 \\
29 & 39 & $1 \cdot 6$ & 96 & 4
\end{tabular}


The nature of these growth-promoting substances has not been established, but their concentration in a magnesium-limited culture of Bacillus subtiiis varied with population density. Thus, when the concentration of magnesium in a culture of B. subtilis was decreased to a value $\left(0.3 \mu \mathrm{g}\right.$. $\mathrm{Mg}^{2+} / \mathrm{ml}$.) such that the steady-state culture population density was about $1 \times 10^{8}$ bacilli/ml., the growth-promoting capacity of the resulting extracellular fluid was decreased correspondingly (Fig. 4). At this lower bacterial concentration small numbers of yeast organisms still could not outgrow the bacilli, but when the culture bacterial population density was decreased further, to $6 \times 10^{6} \mathrm{bacilli} / \mathrm{ml}$. (culture magnesium concentration, $0.15 \mu \mathrm{g} . / \mathrm{ml}$.), proportionately small numbers of the yeast organisms (about $1 \times 10^{7} \mathrm{orgs} / \mathrm{ml}$., initially) now outgrew the bacilli (Table 2) but only slowly. Presumably the very small population of $B$. subtilis organisms could not produce a sufficient concentration of extracellular growth promoting substance(s) to facilitate magnesium uptake by the bacilli to an extent that would allow them to compete effectively with the yeast organisms for the available magnesium.

\section{Table 2. Growth of Bacillus subtilis and Torula utilis at low population densities in a magnesium-limited culture}

The magnesium-limited simple salts medium contained $0.15 \mu \mathrm{g} . \mathrm{Mg}^{2+} / \mathrm{ml}$; $0.1 \mathrm{ml}$. of a $T$. utilis chemostat culture, growing in this low-magnesium medium, was transferred to a $B$. subtilis culture also growing in this medium. The dilution rate was maintained throughout at $0.3 \mathrm{hr}^{-1}$ and the temperature controlled at $33^{\circ}$. The $\mathrm{pH}$ value (uncontrolled) was 6.4 and did not vary.

\begin{tabular}{|c|c|c|c|c|}
\hline \multirow{2}{*}{$\begin{array}{l}\text { Time after } \\
\text { mixing (hr) }\end{array}$} & \multicolumn{2}{|c|}{ No. orgs $/ \mathrm{ml} .\left(\times 10^{-6}\right)$} & \multicolumn{2}{|c|}{$\begin{array}{c}\text { Proportion of } \\
\text { total no. orgs (\%) }\end{array}$} \\
\hline & B. subtilis & T. utilis & B. subtilis & T. utilis \\
\hline 0 & 6 & $<0.1$ & 99 & I \\
\hline 3 & 6 & $<0.1$ & 99 & I \\
\hline 6 & 6 & $<0.1$ & 99 & I \\
\hline 23 & 6 & 0.3 & 95 & 5 \\
\hline 30 & $2 \cdot 2$ & 3.6 & 38 & 62 \\
\hline 49 & $<0.1$ & 9.4 & I & 99 \\
\hline
\end{tabular}

The inability of small numbers of Bacillus subtilis or B. megaterium organisms to outgrow Torula utilis in a simple salts medium did not obtain when magnesium was present in excess of requirement and growth was limited either by the availability of potassium or glucose. In both these cases the bacilli rapidly outgrew the yeast, irrespective of the initial population densities. This finding suggests that the growthpromoting substances were associated specifically with magnesium assimilation and not with cation uptake in general.

\section{DISCUSSION}

The above results provide an explanation for the unpredictable behaviour of some magnesium-limited mixed cultures described here and previously (Tempest et al. 1967); clearly magnesium uptake by both Bacillus subtilis and B. megaterium was facilitated by substances that were secreted into the extracellular fluids when the bacilli were growing under conditions where availability of magnesium limited growth. Since the concentration of these growth-promoting substances varied with population density, so did the ability of the bacilli to compete with other organisms 
in magnesium-limited chemostat mixed cultures. As yet we have no precise knowledge of the nature of these growth-promoting substances but the active material present in a magnesium-limited culture of B. subtilis was found to survive heating at $100^{\circ}$ for $30 \mathrm{~min}$., diffuse through a dialysis sac and separate on a Sephadex column (G-10) with material of molecular weight greater than 700 . Since both Casamino acids and yeast extract promoted growth of $B$. subtilis and B. megaterium in low-magnesium environments (but had no specificity), the growth-promoting substances possibly may be polypeptides or polynucleotides; they do not appear to be teichoic acids. It may be significant that products of RNA degradation are usually, if not invariably, found in extracellular fluids from non-growing suspensions of bacteria (Strange, Dark \& Ness, 196I; Postgate \& Hunter, 1962; Strange, Wade \& Ness, 1963; Dawes \& Ribbons, I964), particularly when the extracellular magnesium concentration is low (Strange \& Hunter, 1967), and nucleotides have been found to be secreted by cultures of B. subtilis growing in defined media (Demain, Burg \& Hendlin, 1965). Also of interest in this connexion is the observation of Byers, Powell \& Lankford (1967) that B. megaterium could secrete a substance (Schizokinen) that actively initiated cell division by chelating iron and facilitating its transport into the cell.

Several workers have observed an effect of population density on the growth of bacteria in both 'batch' and 'continuous' cultures. Bail (1929) proposed the concept of a bacterial space requirement to account for inhibition of growth at high population densities, and Contois (1959) provided evidence that assimilation of $\mathrm{NH}_{4}{ }^{+}$, by Aerobacter aerogenes growing in an $\mathrm{NH}_{4}{ }^{+}$-limited chemostat culture, was less efficient at high population densities than at low ones. On the other hand, Jannasch (1962, I963) showed with carbon-limited chemostat cultures of Spirillum serpens that assimilation of growth-limiting substrate (lactate) was inefficient at low population densities; this he ascribed in part to lack of reducing power in cultures of low population density. Our results suggest, like Jannasch's, that assimilation of growth-limiting substrate (magnesium) was less efficient in the cultures of low population density. Thus, although the method used for determining extracellular magnesium was insufficiently sensitive to detect small changes $\left(<0.05 \mu \mathrm{g} . \mathrm{Mg}^{2+} / \mathrm{ml}\right.$.) in the steady-state growth-limiting substrate concentration, the mixed culture experiments indicated clearly that for Bacillus subtilis and B. megaterium, at least, the relationship between growth-limiting substrate concentration $(s)$ and growth rate $(\mu)$ varied with population density when the availability of magnesium limited growth.

Although the Michaelis-Menten type of equation (I) may describe accurately the relationship between $\mu$ and $s$ in many real chemostat cultures, clearly it is inadequate for cultures in which growth is affected by bacterial products which accumulate in the environment. In such cases a 'population factor' (or, more accurately, a 'population-product factor') must be incorporated in the growth equation. But how equation (I) should be modified to accommodate such a factor is a matter for conjecture, since it is not certain whether its effect is primarily on the saturation constant $\left(K_{s}\right)$ or on the maximum growth rate value $\left(\mu_{\text {max }}\right)$ or on both. Recent work by Dr E. O. Powell (personal communication), using a turbidostat to determine values of $\mu_{\max }$, showed that magnesium-limited Bacillus subtilis organisms secreted a substance (or substances) which increased the maximum growth-rate of $B$. subtilis growing in a low magnesium medium; whether the saturation constant $\left(K_{s}\right)$ was also affected could not be determined. If the growth-promoting substance affected $\mu_{\max }$ only, 
then the simplest relationship that can be derived to accommodate its effect on growth is as follows:

$$
\mu=\mu_{\max }\left(\frac{\mathrm{I}+\lambda p}{\mathrm{I}+p}\right)\left(\frac{s}{K_{s}+s}\right)
$$

where $p$ represents the concentration of extracellular growth-promoting metabolite and $\lambda$ is a constant; $\mu_{\max }$ is defined as the maximum growth rate when $p=0$. Thus, plotting the relationship between $s$ and $\mu$ for magnesium-limited chemostat cultures of $B$. subtilis that differed only in the concentration of magnesium in the input medium (and hence in culture population density and concentration of extracellular growth-promoting substance) would produce a family of curves, similar to those shown in Fig. I, each describing the relationship between $\mathrm{s}$ and $\mu$ at a particular culture population density. At low population densities the concentration of growthpromoting substance would be negligible, and then the relationship between growth rate and growth-limiting substrate concentration given by equation (2) would reduce to that given by equation (I). At high population densities, on the other hand, the concentration of growth-promoting substance would reach a 'saturation' value at which it would exert its maximum effect; thus $(\mathrm{I}+\lambda \mathrm{p}) /(\mathrm{I}+\mathrm{p})$ would approximate to $\lambda$ and the maximum growth rate approach a value equal to $\lambda \mu_{\max }$.

Powell (1958) and Jannasch (I967) concluded that the fate of a contaminant organism in a chemostat culture depended on many factors including the nature of the growth-limiting component of the medium, the dilution rate, temperature and culture $\mathrm{pH}$ value. Our results show that under some conditions at least, the culture population density is a further factor.

We are indebted to Dr E. O. Powell and Mr R. E. Strange for many useful discussions during the progress of this work. We also thank Mr T. H. Dunham for his skilled technical assistance.

\section{REFERENCES}

BaIL, O. (1929). Ergebnisse experimenteller Populationsforschung. Z. ImmunForsch. 6o, I.

Byers, B. R., Powell, M. V. \& LANKFord, C. E. (1967). Iron-chelating hydroxamic acid (Schizo kinen) active in initiation of cell division in Bacillus megaterium. J. Bact. 93, 286.

ContoIs, D. E. (1959). Kinetics of bacterial growth. Relationship between population density and specific growth rate of continuous cultures. J. gen. Microbiol. 21, 40.

Dawes, E. A. \& Ribbons, D. W. (1964). Some aspects of the endogenous metabolism of bacteria. Bact. Rev. 28, 126.

Demain, A. L., Burg, R. W. \& Hendlin, D. (1965). Excretion and degradation of ribonucleic acid by Bacillus subtilis. J. Bact. 89, 640 .

Herbert, D., Elsworth, R. \& Telling, R. C. (1956). The continuous culture of bacteria; a theoretical and experimental study. J. gen. Microbiol. 14, 601.

JANNASCH, H. W. (1962). Bacterial growth at low population densities. Nature, Lond. 196, 496.

JANNASCH, H. W. (1963). Bacterial growth at low population densities (II). Nature, Lond. 197, 1322.

JANNASCH, H. W. (1968). Enrichments of aquatic bacteria in continuous culture. Arch. für Mikrobiol. $59,165$.

MeERs, J. L. \& TempesT, D. W. (I968). Influence of extracellular products on the growth of mixed microbial populations in $\mathrm{Mg}^{2+}$-limited chemostat cultures. J. gen. Microbiol. $\mathbf{5 0}^{\circ}$, iv.

Monod, J. (1950). La technique de culture continue; théorie et applications. Annls Inst. Pasteur, Paris 79, 390.

Postgate, J. R. \& Hunter, J. R. (I962). The survival of starved bacteria. J. gen. Microbiol. 29, 233.

Powell, E. O. (1958). Criteria for the growth of contaminants and mutants in continuous culture. J. gen. Microbiol. 18, 259. 
Powell, E. O. (1965). Theory of the chemostat. Lab. Practice 14, I 145.

STRANGE, R. E. \& HUNTER, J. R. (1967). Effect of magnesium on the survival of bacteria in aqueous suspension. In Microbial Physiology and Continuous Culture, p. I02. London: H.M.S.O.

Strange, R. E., Dark, F. A. \& Ness, A. G. (I96I). The survival of stationary phase cells of Aerobacter aerogenes stored in aqueous suspension. J. gen. Microbiol. 25, 6I.

Strange, R. E., Wade, H. E. \& Ness, A. G. (1963). The catabolism of proteins and nucleic acids in starved Aerobacter aerogenes. Biochem. J. 86, 197.

Tempest, D. W., Dicks, J. W. \& Hunter, J. R. (I966). The interrelationship between potassium, magnesium and phosphorus in potassium-limited chemostat cultures of Aerobacter aerogenes. J. gen. Microbiol. 45, 135.

Tempest, D. W., Dicks, J. W. \& MeErs, J. L. (1967). Magnesium-limited growth of Bacillus subtilis, in pure and mixed cultures, in a chemostat. J. gen. Microbiol. 49, 139.

Tempest, D. W., Hunter, J. R. \& Sykes, J. (1965). Magnesium-limited growth of Aerobacter aerogenes in a chemostat. J. gen. Microbiol. 39, 355. 